OPEN ACCESS

Edited by:

Yan Zhang,

Zhejiang University, China

Reviewed by:

Ye-Tang Pan,

Beijing Institute of Technology, China

Juan Li,

Ningbo Institute of Materials Technology and Engineering (CAS),

China

*Correspondence:

Chunzhuang Yang

zhihe@zhpolymer.com

Specialty section:

This article was submitted to

Polymeric and Composite Materials,

a section of the journal

Frontiers in Materials

Received: 21 May 2021

Accepted: 21 June 2021

Published: 19 July 2021

Citation:

Yang C and Shao S (2021) Rigid

Polyurethane Foams Containing Modified Ammonium Polyphosphate Having Outstanding Charring Ability

and Increased Flame Retardancy.

Front. Mater. 8:712809.

doi: 10.3389/fmats.2021.712809

\section{Rigid Polyurethane Foams Containing Modified Ammonium Polyphosphate Having Outstanding Charring Ability and Increased Flame Retardancy}

\author{
Chunzhuang Yang * and Shuiyu Shao \\ Hangzhou Zhihe New Material Co., Ltd., Zhejiang, China
}

Ammonium polyphosphate (APP) with different polymerization degrees were modified by a novel phosphorus-containing organosilicon compound (PCOC), and the products obtained were coded as MAPP-30 and MAPP-1000. Then they were applied to prepare flame-retardant rigid polyurethane foam (RPUF) separately. The impact of modified APP (MAPP) on the flame-retardant properties of RPUF was investigated by the limited oxygen index (LOI) test, horizontal burning test, and cone calorimeter test. The morphologies of the char residues were observed by SEM. Furthermore, the mechanical properties of RPUF composites were measured by the compressive strength test. The results showed that whether the degree of polymerization of MAPP is 30 or 1000, they both had greater charring ability and better flame-retardant properties than unmodified APP. The residual char yield of RPUF/MAPP-30 (37.3\%) and RPUF/MAPP-1000 (36.5\%) were both significantly higher than RPUF/APP-30 (22.8\%) and RPUF/APP-1000 (24.9\%). The peak heat release rate value of RPUF/MAPP-30 was 29.9\% lower than that of RPUF/ APP-30, and the drop of RPUF/MAPP-1000 was 50.9\% compared to RPUF/APP-1000. Moreover, the total heat release of RPUF/MAPP-1000 $\left(9.7 \mathrm{MJ} / \mathrm{m}^{2}\right)$ was much lower than that of RPUF/MAPP-30 $\left(11.3 \mathrm{MJ} / \mathrm{m}^{2}\right)$. In summary, MAPP-1000 has the best flameretardant properties among all RPUF composites. In addition, the results also showed that flame-retardant performance and the mechanical properties dramatically decreased with the increase in the addition of MAPP-1000, and the RPUF composite had the best comprehensive performance with $20 \%$ content of MAPP-1000.

Keywords: rigid polyurethane foam, ammonium polyphosphate, surface modification, flame retardancy, charring ability

\section{INTRODUCTION}

Rigid polyurethane foam (RPUF), a polymer material, is widely used in the fields of insulation, packaging material, construction panels, and furniture due to its excellent properties, which include low heat conductivity, low density, high strength-to-weight ratio, and aging resistance (Yang et al., 2015; Yang et al., 2017; Yang et al., 2019). Nevertheless, RPUF is highly flammable and easily ignitable due to its unique chemical structure (Levchik and Weil, 2004). Moreover, it generates a large amount of heat and releases some toxic gases during combustion, and the fire hazard greatly endangers the safety and health of humans, significantly limiting its application in many fields (Chen et al., 2019a). Therefore, it is essential to develop excellent flame retardant RPUF materials. 
The traditional flame-retardant treatment of polyurethane foam is the addition of halogenated flame retardants (Shaw et al., 2010). However, most of the halogenated flame retardants had high environmental persistence and harmful bioaccumulation. With the increasing awareness of environmental protection, halogen-free flame retardants (Yang et al., 2009; Sypaseuth et al., 2017; Zheng et al., 2017; Bhoyate et al., 2019; Li et al., 2019a; Chen et al., 2020a) have been developed and applied in flame-retardant RPUF materials due to their advantages of low-smoke, low-toxicity, and high-efficiency.

At present, organic-inorganic hybrid flame retardants (Qin et al., 2015; Chen et al., 2019b) have been widely used and deeply studied due to their good compatibility with polymers and high flame retardant efficiency. Intumescent flame retardants (Camino et al., 1989; Xi et al., 2016; Yuan et al., 2016a; Zhang et al., 2020a) and phosphorus-containing compounds (Liu et al., 2016; Yuan et al., 2016b; Zhang et al., 2020a) such as ammonium polyphosphate (APP), dimethyl methylphosphonate (DMMP), triethyl phosphate, and aluminum hypophosphite draw people's attention due to their low toxicity and high efficiency. They have been widely used in many polymer-based materials. As a typical phosphorusand nitrogen-containing halogen-free flame retardant, APP has been widely used in many polymers including RPUF (Deodhar et al., 2011; Hazer et al., 2019; Wu et al., 2019). The degree of polymerization is an important factor affecting the flame retardancy of APP and also affects the compatibility between APP and the polymer matrix. There are six crystal types of APP, of which type I and type II are commonly used (Watanabe et al., 2000). The degree of polymerization of APP (type I) is usually less than 100, while that of APP (type II) is as high as 1,000 or more. APP with a high degree of polymerization is widely used in materials and coatings. In addition, the poor dispersibility and compatibility of APP with the RPUF matrix result in a low flame-retardant efficiency (Chen et al., 2017a). Therefore, it is necessary to modify APP to improve its flame-retardant efficiency in RPUF. At present, the main methods to solve this problem are microencapsulation or surface treatment with the silane coupling agent (Qin et al., 2015; Xu et al., 2015; Zhang et al., 2020b).

In our previous study, a novel phosphorus-containing organosilicon compound (PCOC) was synthesized and characterized (Chen et al., 2018). In our research, APP with different polymerization degrees (APP-30 and APP-1000) was modified by PCOC, and the products obtained were coded as MAPP-30 and MAPP-1000. The effect of MAPP-30 and MAPP-1000 on the key performance parameters of RPUF materials, such as oxygen index, thermal stability, and cone calorimetry, were explored and compared. The morphologies of residue char were observed, and the mechanical properties of RPUF composites were also investigated. In addition, the effect of the amount of MAPP-1000 on the performance of RPUF was also studied.

\section{EXPERIMENT}

\section{Materials}

Phenylphosphoryl dichloride (BPOD) was purchased from Shanghai Macklin Biochemical Co., Ltd. Low molecular

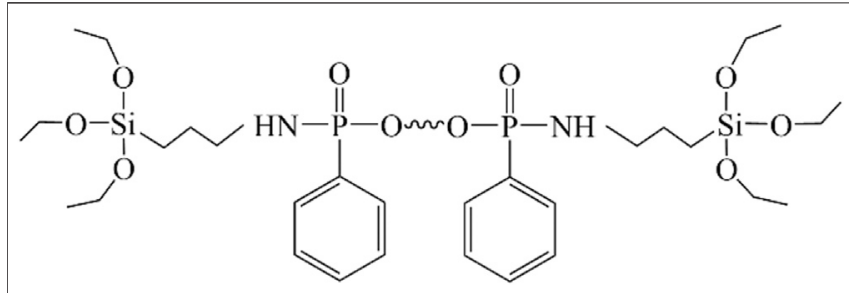

FIGURE 1 | Chemical structure of PCOC.

weight polyether polyol (DP400) was supplied by Kukdo Chemical Co., Ltd. 3-aminopropyltriethoxysilane (KH-550) was supplied by Sinopharm Chemical Reagent Co., Ltd. 1,4Dioxane was supplied by Tianjin Fuchen Chemical Reagents Factory. Tetrahydrofuran (THF) was purchased from Sinopharm Chemical Reagent Co., Ltd. Triethylamine was purchased from Tianjin Kwangfu Fine Chemical Industry Research Institute. APP-30 $(30<n<50)$ and APP-1000 $(n>1,000)$ were purchased from Hangzhou JLS Flame Retardants Chemical Co., Ltd. Premixed polyether polyol (ELASTOSPRAY 81305) was purchased from the German BASF Company (viscosity at $25^{\circ} \mathrm{C}$ : $640 \mathrm{mPa} \times \mathrm{s}$; hydroxyl value: $320 \pm 10 \mathrm{mg} \mathrm{KOH} / \mathrm{g}$ ). Polyisocyanate (PAPI, 44V20) was purchased from Covestro Polymers (China) Co., Ltd. (-NCO\%: 30; monomer methylene diphenyl diisocyanate (MDI)\%: 52).

\section{Synthesis of Phosphorus-Containing Organosilicon Compound}

PCOC was synthesized according to the methods reported in our previous literature (Chen et al., 2018); the chemical structure is shown in Figure 1. Moreover, in this study, a small amount of ammonia water was added to the PCOC at room temperature, and then the $\mathrm{PH}$ value was tested after stirring for a while. The process was repeated until the $\mathrm{PH}$ value of PCOC was neutral. Finally, the surface treatment agent PCOC was obtained after the vacuum distillation process.

\section{Preparation of MAPP-30}

$12 \mathrm{~g}$ of PCOC and $88 \mathrm{ml}$ of anhydrous ethanol were added into a $250 \mathrm{ml}$ three-necked round-bottom glass flask, and then $11 \mathrm{ml}$ distilled water was added in drops into the flask. After the dripping was completed, the mixture was stirred at $25^{\circ} \mathrm{C}$ for $15 \mathrm{~min}$ to derive homogeneous PCOC dispersion (the volume ratio of $\mathrm{PCOC}$, water, and anhydrous ethanol is 1 : 1:8). Simultaneously, $40 \mathrm{~g}$ APP-30 and $200 \mathrm{ml}$ anhydrous ethanol were introduced in a $500 \mathrm{ml}$ three-neck flask, and ultrasonic dispersion was performed at $40^{\circ} \mathrm{C}$ for $40 \mathrm{~min}$. Afterward, the hydrolyzed PCOC was added to the APP suspension at a rate of 2-3 drops per second while maintaining the temperature at $40^{\circ} \mathrm{C}$. The latest mixture was slowly heated to $60^{\circ} \mathrm{C}$, and the reaction was allowed to proceed at $60^{\circ} \mathrm{C}$ for $3 \mathrm{~h}$. After the reaction, the solvent in the reaction mixture was removed by vacuum distillation. Finally, 


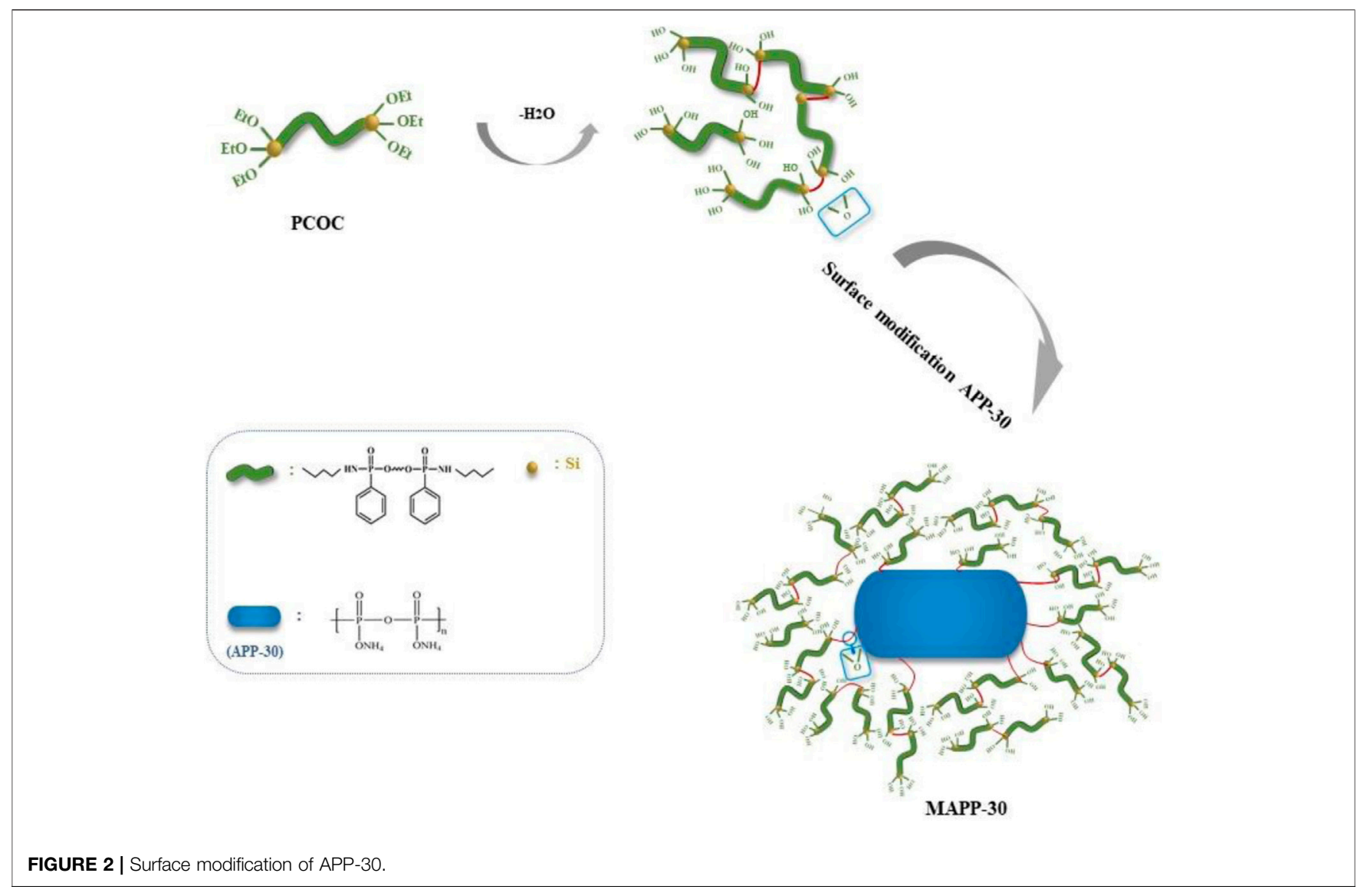

TABLE 1 | Formulas compositions of RPUF composites.

\begin{tabular}{|c|c|c|c|c|c|c|c|}
\hline Sample & Polyether polyol (g) & PAPI (g) & $\mathrm{H}_{2} \mathrm{O}(\mathrm{g})$ & APP-30 (g) & APP-1000 (g) & MAPP-30 (g) & MAPP-1000 (g) \\
\hline Pure RPUF & 100 & 100 & 1 & 0 & 0 & 0 & 0 \\
\hline RPUF/APP-30 & 100 & 100 & 2.5 & 40 & 0 & 0 & 0 \\
\hline RPUF/APP-1000 & 100 & 100 & 2.5 & 0 & 40 & 0 & 0 \\
\hline RPUF/MAPP-30 & 100 & 100 & 2.5 & 0 & 0 & 40 & 0 \\
\hline RPUF/MAPP-1000 & 100 & 100 & 2.5 & 0 & 0 & 0 & 40 \\
\hline RPUF/25MAPP-1000 & 100 & 100 & 2.5 & 0 & 0 & 0 & 50 \\
\hline RPUF/30MAPP-1000 & 100 & 100 & 2.5 & 0 & 0 & 0 & 60 \\
\hline
\end{tabular}

the system was distilled under pressure, and the product was then dried in a vacuum oven at $85^{\circ} \mathrm{C}$ for $8 \mathrm{~h}$. The final product was referred to as MAPP-30. The schematic process of MAPP30 is shown in Figure 2. MAPP-1000 was synthesized according to the methods reported in our previous literature (Chen et al., 2018). MAPP-30 was obtained by using PCOC-modified APP-30, and MAPP-1000 was obtained by using PCOC-modified APP-1000.

\section{Preparation of Flame-Retardant RPUF Composites}

The flame-retardant RPUF composites (FR-RPUFs) were prepared by free-foaming in our laboratory. The compositions of FR-RPUFs are listed in Table 1. Polyether polyol and MAPP (or APP) was first added into a container according to the ratio with stirring to form a uniform mixture. PAPI was immediately added into the same container, followed by stirring at 3,000-4,000 rpm for $10-15 \mathrm{~s}$ to obtain the latest mixture. After the foam was prepared, it demanded for $24 \mathrm{~h}$ curing. Finally, after curing, the foam was cut to prepare standard size samples, accordingly, for testing.

\section{Characterizations}

The molecular structures of APP-30 and MAPP-30 were characterized by Fourier transform infrared (FTIR) spectroscopy on a Nicolet iN10MX spectrometer (Nicolet Instrument Company, Madison, Wisconsin, United States) over the wavenumber range from 500 to $4,000 \mathrm{~cm}^{-1}$ with 32 scans. The test specimens were prepared by the potassium bromide-disk technique. 


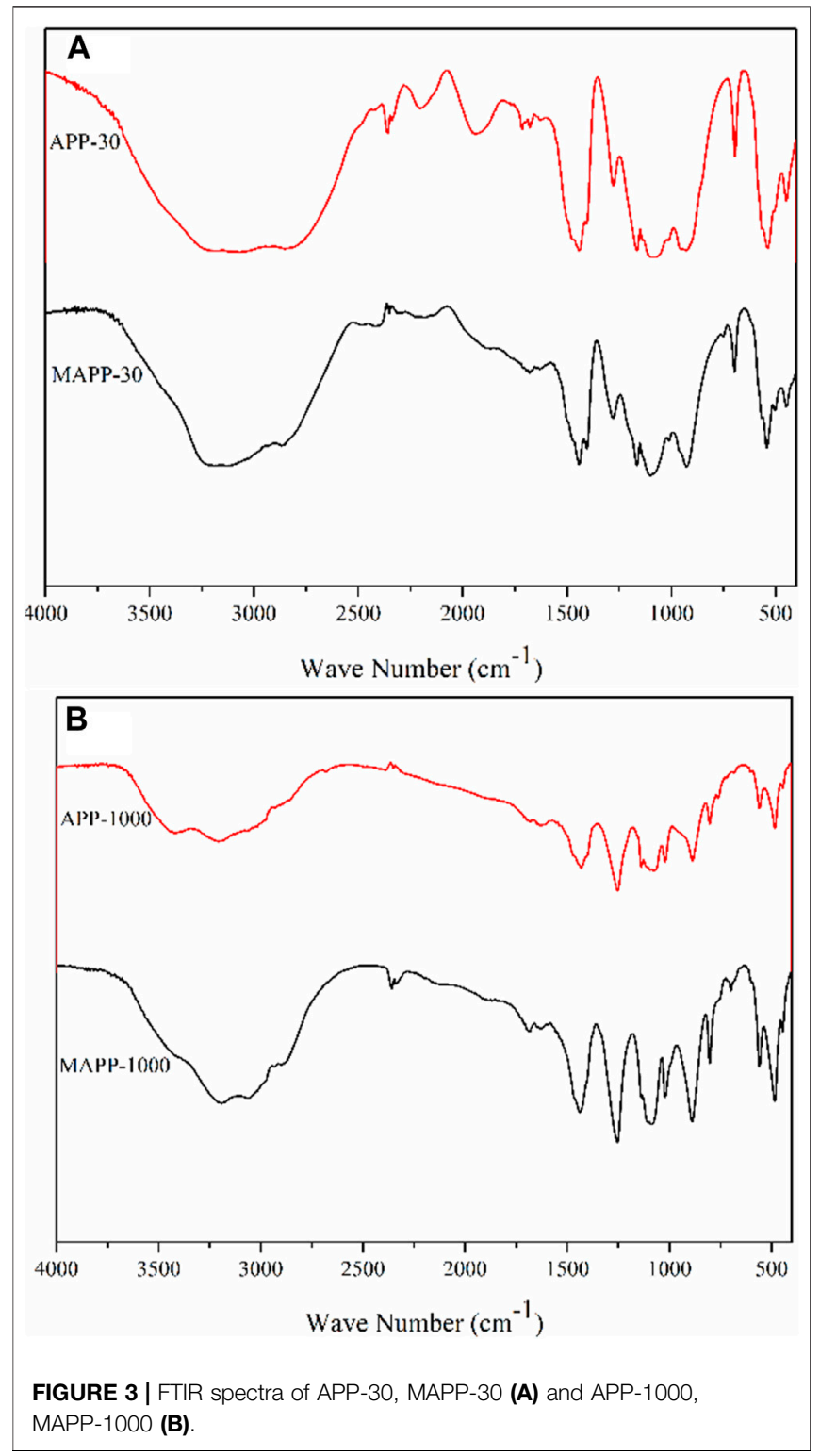

The thermal properties of RPUF composites were characterized by thermogravimetric analysis (TGA) measurements performed using a Q50 IR (TA Instruments, United States) thermal gravimetric analyzer. About $4 \mathrm{mg}$ of the sample was placed in an alumina crucible and heated from 50 to $800^{\circ} \mathrm{C}$ at a rate of $20^{\circ} \mathrm{C} / \mathrm{min}^{-1}$ under $\mathrm{N}_{2}$ atmosphere.

The limiting oxygen index (LOI) values were measured with a Dynisco LOI instrument (Fire Testing Technology (FTT), United Kingdom) according to ASTM D 2863-97 with a dimension specimen of $100.0 \mathrm{~mm} \times 10.0 \mathrm{~mm} \times 10.0 \mathrm{~mm}$.

The horizontal burning test was performed on an FTT 0082 instrument (Fire Testing Technology Ltd., United Kingdom) in accordance with ISO 09772-2001. The dimension of the samples was measured as $150.0 \mathrm{~mm} \times 50.0 \mathrm{~mm} \times 13.0 \mathrm{~mm}$ (length $\times$ width $\times$ thickness).
The fire behavior was characterized by using an FTT cone calorimeter (Fire Testing Technology Ltd., United Kingdom) at a heat flux of $50 \mathrm{~kW} / \mathrm{m}^{2}$ according to ISO 5660 , and the dimension of the samples was $100.0 \mathrm{~mm} \times 100.0 \mathrm{~mm} \times 3.0 \mathrm{~mm}$. The measurement for each sample was repeated thrice, and the typical cone calorimetry data were reproducible within $\pm 10 \%$.

The micromorphology of the residues of FR-RPUFs after the cone calorimeter test was observed by using a Phenom Pro scanning electron microscope (SEM, Phenom World, Netherlands) under high vacuum conditions with an accelerating voltage of $5 \mathrm{kV}$.

The compressive properties of the FR-RPUFs were characterized by a CMT6104 universal testing machine (Sheng Zhen, China) according to ISO 844-1787. The measurement for each sample was repeated three times at least to obtain average values. The dimension of each specimen was $50.0 \mathrm{~mm} \times 50.0 \mathrm{~mm}$ $\times 50.0 \mathrm{~mm}$ (length $\times$ width $\times$ thickness).

\section{RESULTS AND DISCUSSION}

\section{Characterization of Modified APP}

Figure 3 shows the FTIR spectra of APP-30 and APP-1000 before and after modification. It could be seen from Figure $\mathbf{3 A}$ that typical peaks of APP-30 mainly include $\mathrm{N}-\mathrm{H}\left(3,250 \mathrm{~cm}^{-1}\right.$, $\left.1,400 \mathrm{~cm}^{-1}\right), \quad \mathrm{P}=\mathrm{O} \quad\left(1,270 \mathrm{~cm}^{-1}\right), \quad$ and $\mathrm{P}-\mathrm{O}-\mathrm{P} \quad\left(850 \mathrm{~cm}^{-1}\right)$. Compared with the spectrum of APP-30, the absorption peaks of Si-O-C groups can be clearly observed at $1,000 \sim 1,100 \mathrm{~cm}^{-1}$ for MAPP-30, and the stretching vibration peaks of the $\mathrm{C}-\mathrm{H}$ groups of aromatic rings also can be found at $2,960 \sim 2,850 \mathrm{~cm}^{-1}$. From the results, it can be concluded that PCOC has been successfully grafted onto the surface of APP-30. As shown in Figure 3B, the most characteristic peaks of APP-1000 and PCOC still existed in MAPP-1000. The typical characteristic peaks of APP-1000 are at $3,200(\mathrm{~N}-\mathrm{H}), 1,250(\mathrm{P}=\mathrm{O})$, and $830 \mathrm{~cm}^{-1}(\mathrm{P}-\mathrm{O}-\mathrm{P})$. The peaks at $2,960 \sim 2,850 \mathrm{~cm}^{-1}$ were assigned to the characteristic peaks of $\mathrm{C}-\mathrm{H}$ stretching vibrations, which came from PCOC. The peaks at $1,480 \mathrm{~cm}^{-1}$ is due to $\mathrm{C}-\mathrm{C}$ of aromatic rings skeletal vibrations. Moreover, the stronger absorption peaks emerging at about $1,100 \mathrm{~cm}^{-1}$ corresponded to the Si-O-C stretching vibrations. The above information indicates that the modification of APP1000 was also successful.

\section{Thermal Stability of Ammonium Polyphosphate and Modified APP Flame-Retardant Additives}

The thermal stability and amount of residue at high temperature are essential indicators to evaluate flame retardants (Chen et al., 2017b). A comparative thermal degradation behavior of unmodified and modified APP under $\mathrm{N}_{2}$ can further analyze the thermal decomposition behavior of MAPP. The thermogravimetric analysis (TGA) and derivative thermogravimetric (DTG) curves of APP-30, APP-1000, MAPP30, and MAPP-1000 under $\mathrm{N}_{2}$ are shown in Figure 4. The typical data are summarized in Table 2 , such as the initial decomposition 


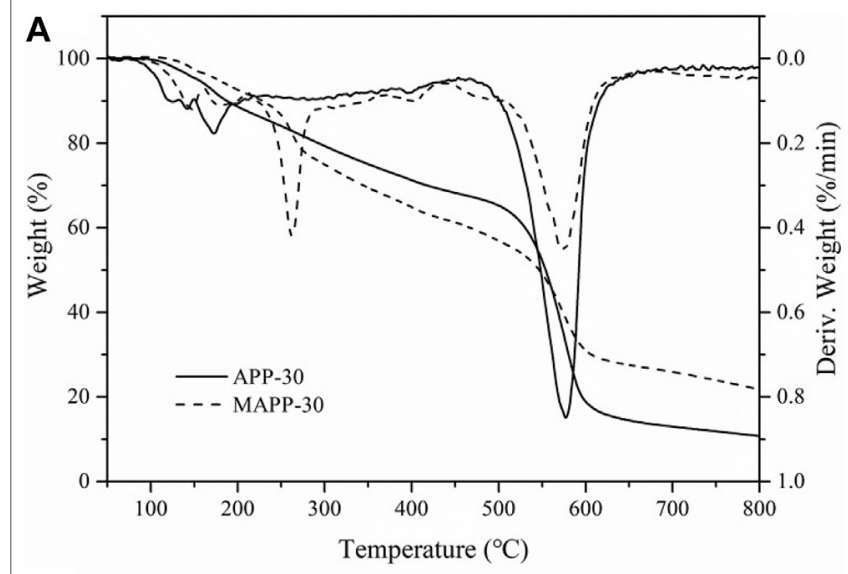

B

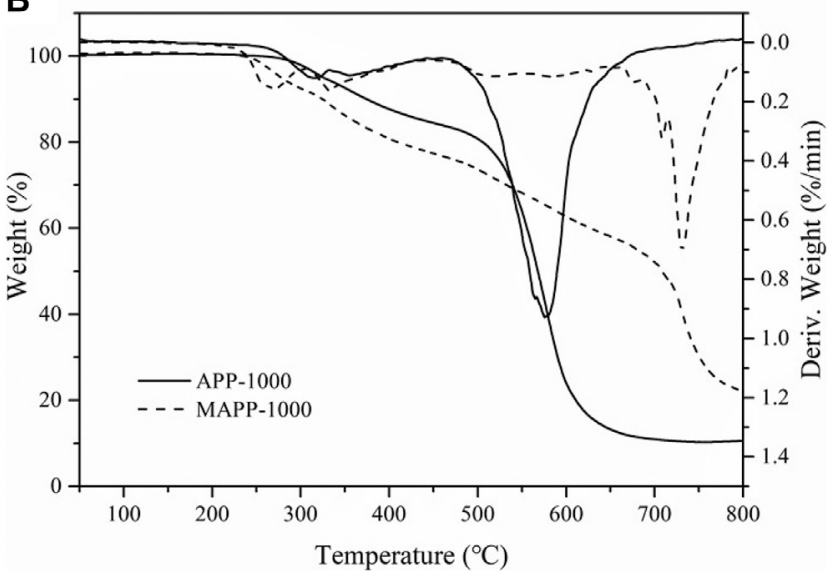

FIGURE 4 | TGA and DTG curves of APP-30, MAPP-30 (A), and APP1000, MAPP-1000 (B).

TABLE 2 | TGA Data of APP-30, MAPP-30, APP-1000, and MAPP-1000.

\begin{tabular}{lccc} 
Samples & $\boldsymbol{T}_{\mathbf{d}, \mathbf{5} \%}\left({ }^{\circ} \mathbf{C}\right)$ & $\boldsymbol{T}_{\max }\left({ }^{\circ} \mathbf{C}\right)$ & $\begin{array}{c}\text { Residuals } \\
\mathbf{a t ~} \mathbf{8 0 0} \mathbf{~}^{\mathbf{C}} \text { (wt\%) }\end{array}$ \\
\hline APP-30 & 154.7 & 578.2 & 10.8 \\
MAPP-30 & 177.5 & 575.1 & 21.8 \\
APP-1000 & 325.7 & 578.8 & 10.9 \\
MAPP-1000 & 277.9 & 732.4 & 22.0 \\
\hline
\end{tabular}

temperature $\left(T_{\mathrm{d}, 5 \%}\right)$, the maximum decomposition temperature $\left(T_{\max }\right)$, and residuals at $800^{\circ} \mathrm{C}$.

According to the TGA curves, the thermal degradation of APP-30 and APP-1000 both involve two stages in the nitrogen atmosphere. The first degradation stage occurred before $500^{\circ} \mathrm{C}$; the evolved products at this stage were mainly $\mathrm{NH}_{3}, \mathrm{H}_{2} \mathrm{O}$, and phosphoric acid. Then, the second degradation stage occurred above $500^{\circ} \mathrm{C}$; polymetaphosphate and polyphosphoric acid were formed. The $T_{\max }$ of APP-30 $\left(578.2^{\circ} \mathrm{C}\right)$ in the second stage was similar to that of APP-1000 $\left(578.8^{\circ} \mathrm{C}\right)$. In contrast, the $T_{\mathrm{d}, 5 \%}$ of APP-30 $\left(154.7^{\circ} \mathrm{C}\right)$ was lower than that of APP-1000 $\left(325.7^{\circ} \mathrm{C}\right)$,
TABLE 3 | Results of LOI test and horizontal burning test for RPUF composites.

\begin{tabular}{lcc}
\hline Samples & LOI (\%) & Horizontal burning rating \\
\hline Pure RPUF & 20.6 & $\mathrm{HBF}$ \\
RPUF/APP-30 & 24.2 & $\mathrm{HF}-1$ \\
RPUF/APP-1000 & 24.6 & $\mathrm{HF}-1$ \\
RPUF/MAPP-30 & 24.2 & $\mathrm{HF}-1$ \\
RPUF/MAPP-1000 & 24.5 & $\mathrm{HF}-1$ \\
RPUF/25MAPP-1000 & 24.9 & $\mathrm{HF}-1$ \\
RPUF/30MAPP-1000 & 24.9 & $\mathrm{HF}-1$
\end{tabular}

and the weight loss of APP-30 is $40 \%$ in the first stage, while APP1000 is $20 \%$. Through literature review, we find that the initial decomposition temperature of APP with a low degree of polymerization is about $150-250^{\circ} \mathrm{C}$, which is due to the short molecular chain (Hazer et al., 2019).

MAPP-30 was the final product of APP-30 modified by PCOC, and it showed three decomposition stages. As can be seen from Table 2, the $T_{\mathrm{d}, 5 \%}$ of MAPP-30 $\left(177.5^{\circ} \mathrm{C}\right)$ was higher than APP-30 $\left(154.7^{\circ} \mathrm{C}\right)$, indicating the better thermal stability of MAPP-30 after the modification of PCOC. The reason for higher initial decomposition temperature of MAPP-30 than APP-30 is that the initial decomposition temperature of PCOC is above $200^{\circ} \mathrm{C}$. Combined with Table 2, the second stage was from 200 to $300^{\circ} \mathrm{C}$, attributing to the decomposition of PCOC. The third degradation stage occurred above $500^{\circ} \mathrm{C}$, which was the main degradation stage of MAPP-30. The $T_{\max }$ in this stage was $575.1^{\circ} \mathrm{C}$, which was similar with APP-30, indicating the same degradation process of APP-30 and MAPP-30 in this stage.

MAPP-1000 also showed three degradation processes. From 200 to $400^{\circ} \mathrm{C}$, APP decomposed and released $\mathrm{NH}_{3}$ and $\mathrm{H}_{2} \mathrm{O}$. PCOC also decomposed at this stage. The $T_{\mathrm{d}, 5 \%}$ of MAPP- 1000 $\left(277.9^{\circ} \mathrm{C}\right)$ was lower than that of APP-1000 $\left(325.7^{\circ} \mathrm{C}\right)$, which was due to the induced decomposition effect of PCOC to promote the earlier decomposition of MAPP-1000. From 500 to $600^{\circ} \mathrm{C}$, MAPP-1000 had a slight weight loss, but APP-1000 mainly decomposed during this temperature range. It indicated that the degradation process of MAPP-1000 was not the same as APP-1000. MAPP-1000 mainly decomposed in the third stage $\left(700-800^{\circ} \mathrm{C}\right)$. The $T_{\max }$ for MAPP-1000 $\left(732.4^{\circ} \mathrm{C}\right)$ was significantly higher than that of APP-1000 $\left(578.8^{\circ} \mathrm{C}\right)$. It demonstrated that more stable structures, which may be produced by the reaction between PCOC and APP-1000, were formed during high temperatures. It is noteworthy that the residual char at $800^{\circ} \mathrm{C}$ of MAPP-30 and MAPP-1000 were $21.8 \mathrm{wt} \%$ and $22.0 \mathrm{wt} \%$, which were obviously higher than APP-30 (10.8 wt\%) and APP-1000 (10.9 wt\%), respectively. In summary, the $T_{\mathrm{d}, 5 \%}, T_{\max }$, and char yield of MAPP-1000 were higher than that of MAPP-30, indicating that MAPP-1000 had a better thermal stability.

\section{Flame-Retardant Performance of RPUF Composites}

Horizontal burning and LOI tests are commonly used to characterize the flammability of RPUF composites (Jing et al., 

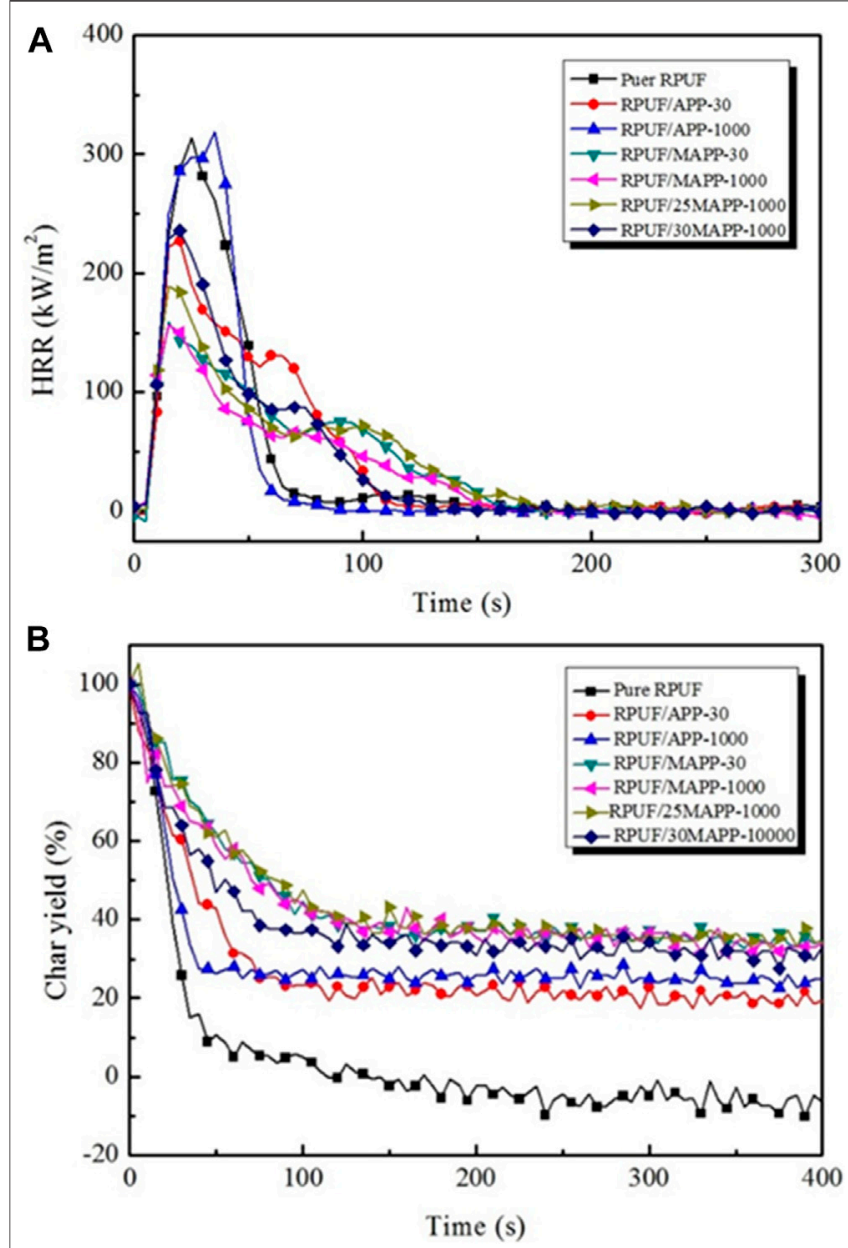

FIGURE 5 | HRR (A) and Char yield (B) curves of FR-RPUFs. Macroscopic and Microscopic Morphologies of Residual Char.

2018; Li et al., 2019b; Sai et al., 2021). Table 3 shows the LOI values and the results of the horizontal burning test. It could be seen that pure RPUF had an LOI value of only $20.6 \%$ and reached $\mathrm{HBF}$ is a level of UL 94 horizontal burning rating. As could be seen from Table 3, the LOI values were almost unchanged when MAPP-30 or MAPP-1000 were added into RPUF alone compared with that of RPUF/APP-30 or RPUF/APP-1000. The LOI value of RPUF/MAPP- 1000 was $24.5 \%$. With the increase in the addition of MAPP-1000, the LOI values of RPUF composites containing MAPP-1000 had a slight increase. The LOI values of RPUF/ 25MAPP-1000 and RPUF/30MAPP-1000 were both 24.9\%. In addition, the results of the horizontal burning test show that RPUF/APP systems and RPUF/MAPP systems can all reach HF-1 rating.

The cone calorimeter test is a widely applied technique in evaluating the fire performance (Bhoyate et al., 2018). In this work, the flame-retardant behavior of MAPP on RPUF was further evaluated by means of the cone calorimeter test. The heat release rate (HRR) and char yield curves for FR-RPUFs are presented in Figure 5. In addition, partial data, such as the peak heat release rate (PHRR), average value of effective heat of combustion (av-EHC), total heat release (THR), total smoke release (TSR), average value of mass loss rate (av-MLR), and char yield, are listed in Table 4.

Figure $\mathbf{5 A}$ gives the HRR curves of pure RPUF and RPUF composites with different flame retardants. It could be seen that pure RPUF burns rapidly after ignition and its PHRR value is $310 \mathrm{~kW} / \mathrm{m}^{2}$. When APP-30 is added into RPUF, the HRR of RPUF/APP-30, the PHRR $\left(227.4 \mathrm{~kW} / \mathrm{m}^{2}\right)$ is lower than that of pure RPUF. After the addition of untreated APP into RPUF, APP-30 decreased the PHRR, and the addition of APP-1000 increased the peak heat release rate slightly. This may be due to the poor dispersion of APP in RPUF due to the increase in the degree of polymerization (Zhang et al., 2018). The PHRR value of RPUF/MAPP-30 is $158.6 \mathrm{~kW} / \mathrm{m}^{2}$, which was decreased by $48.9 \%$ compared with RPUF/APP-30. The similar phenomenon also occurred in RPUF/MAPP-1000, and the drops were $30 \%$. It is obvious that the PHRR values of RPUF composites containing modified APP were all reduced, as compared to the RPUF/APP systems. The reduction of PHRR values may be related to the formation of high amount of table char residues, which can be proved by the char yields data in Table 4. Moreover, the PHRR value of RPUF gradually increases as MAPP-1000 loading increases, which may be due to the weak protective effect of the char layer.

After the modification of APP by PCOC, the THR of all RPUF/ MAPP composites decreased. In contrast, the THR values of RPUF/MAPP-1000 $\left(9.7 \mathrm{MJ} / \mathrm{m}^{2}\right)$ was lower than that of RPUF/ MAPP-30 $\left(11.3 \mathrm{MJ} / \mathrm{m}^{2}\right)$. When the addition of MAPP-1000 increased to 25 and $30 \%$ from $20 \%$, the THR values of RPUF/ 25MAPP-1000 and RPUF/30MAPP-1000 were increased compared with RPUF/MAPP-1000. This was because of the poor compatibility between excessive MAPP-1000 and RPUF matrix, which reduced the flame retardancy of the RPUF composites.

Figure 5B shows the char yield curves of the RPUF composite samples. For pure RPUF, the amount of char yield was only $12.2 \%$. Additionally, the char yields for RPUF/APP-30 and RPUF/APP-1000 was 22.8 and 24.9\%, while the values for RPUF/MAPP-30 and RPUF/MAPP-1000 were 37.3 and $36.5 \%$, respectively, indicating an efficient char forming ability of MAPP for RPUF. It was worth noting that in the RPUF/MAPP systems, when the amount of MAPP was only $20 \%$, the char yield of the composites was the highest. The protective char layer formed during the combustion of RPUF/MAPP can isolate the RPUF from the flame and prevent the heat transfer, thus leading to better flame retardancy. The more char residue formed during the combustion leads to the better barrier protection.

The change in the residual char also affects the TSR values of RPUF. The TSR value of pure RPUF is $505.7 \mathrm{~m}^{2} / \mathrm{m}^{2}$; after the addition of APP, the TSR values increase obviously. The reason is APP can promote the carbonization of RPUF, leading to incomplete combustion of RPUF and thus yielding more smoke. In addition, TSR of RPUF/MAPP systems were much lower than RPUF/APP systems, indicating that the modification procedure by PCOC can lock more combustion products in the condensed phase and effectively inhibit the release of smoke 
TABLE 4 | Results of cone calorimeter test for RPUF composites.

\begin{tabular}{|c|c|c|c|c|c|}
\hline Samples & Pk-HRR (kW/m²) & Av-EHC (MJ/kg) & THR (MJ/m²) & $\operatorname{TSR}\left(\mathrm{m}^{2} / \mathrm{m}^{2}\right)$ & $\begin{array}{c}\text { Char yields } \\
(\%)\end{array}$ \\
\hline Pure RPUF & 310 & 19.4 & 12.2 & 506 & 12.2 \\
\hline RPUF/APP-30 & 227 & 16.2 & 12.7 & 595 & 22.8 \\
\hline RPUF/APP-1000 & 318 & 15.7 & 11.2 & 642 & 24.9 \\
\hline RPUF/MAPP-30 & 159 & 16.7 & 11.3 & 390 & 37.3 \\
\hline RPUF/MAPP-1000 & 156 & 17.1 & 9.7 & 326 & 36.5 \\
\hline RPUF/25MAPP-1000 & 189 & 17.2 & 12.1 & 454 & 35.1 \\
\hline RPUF/30MAPP-1000 & 236 & 19.0 & 11.7 & 543 & 34.2 \\
\hline
\end{tabular}
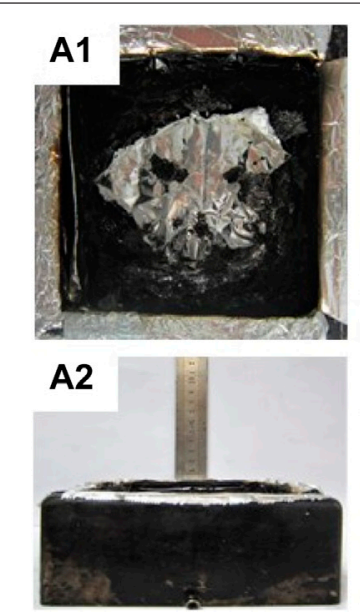
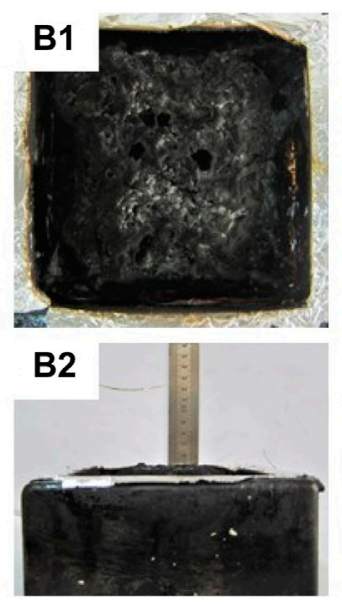
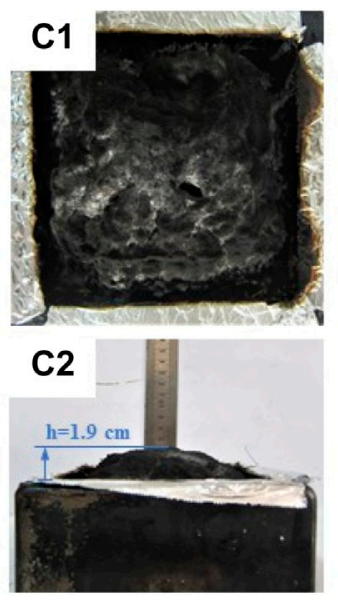
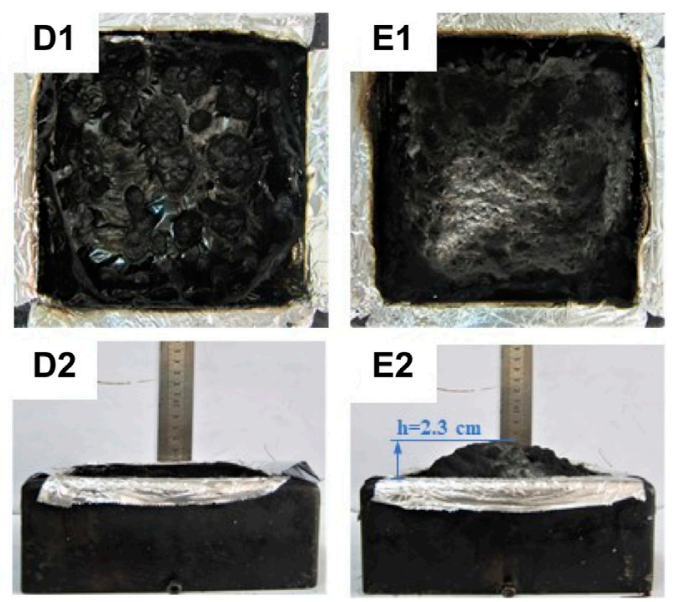

FIGURE 6 | Residual char digital photos of Pure RPUF (A), RPUF/APP-30 (B), RPUF/MAPP-30 (C), RPUF/APP-1000 (D) and RPUF/MAPP-1000 (E).

(Bhoyate et al., 2018). For pure RPUF, the value of TSR is $505.7 \mathrm{~m}^{2} / \mathrm{m}^{2}$ and decreases significantly to $326.0 \mathrm{~m}^{2} / \mathrm{m}^{2}$ for RPUF/MAPP-1000, which can be interpreted as formation of the dense carbon layer; the total amount of smoke is decreased during the combustion process. Moreover, the av-EHC values of RPUF/APP-30, RPUF/APP-1000, RPUF/MAPP-30, and RPUF/ MAPP-1000 are $16.2 \mathrm{MJ} / \mathrm{kg}, 15.7 \mathrm{MJ} / \mathrm{kg}, 16.7 \mathrm{MJ} / \mathrm{kg}$, and $17.1 \mathrm{MJ} / \mathrm{kg}$, a drop of $16.5,19.1,13.9$, and $11.8 \%$ than that of pure RPUF $(19.4 \mathrm{MJ} / \mathrm{kg})$. It can be deduced that APP exhibits good flame-retardant efficiency in the gas phase. Additionally, the av-EHC values of RPUF/MAPP samples were slightly higher than that of RPUF/APP, indicating the RPUF/MAPP composites worked mainly through condensed phase. In conclusion, MAPP-1000 could exert a better flame retardancy with a proper addition (20\%) in RPUF.

In order to further explore the effect of the polymerization degree of APP on the flame retardant performance of MAPP in RPUF, the morphology of residual char after cone calorimetry was analyzed in detail. Figure 6 showed the digital photos of RPUF composites after the cone calorimeter test. As can be seen from Figure 6, there was almost no residual char after the combustion of pure RPUF. As can be seen from Figures 6B,C, the char layers of both RPUF/APP-30 and RPUF/APP-1000 had no height. Compared with RPUF/APP-1000, the char layer of RPUF/APP-30 was more complete, but its surface was cracked. Moreover, the char layer of RPUF/APP-1000 presented an "island structure," indicating that the dispersion of APP-1000 in the matrix was poor. Compared with RPUF/APP systems, the residual char of RPUF/MAPP systems were significantly increased, and the intumescent phenomenon of the char layer was also observed. Compared with Figures 6D,E, it showed that the residual char of RPUF/MAPP-1000 was more than that of RPUF/APP-1000, and the height of RPUF/MAPP-1000 carbon layer was the largest. The char layer generated by RPUF/MAPP1000 was more complete and compact with no obvious cracks on its surface and presented the morphology of the intumescent char layer, which indicated that RPUF/MAPP-1000 had a better charforming ability. The formation of the intumescent char layer was due to uniform dispersion of APP after coating on the one hand and the surface treatment agent PCOC acting as the carbon source on the other hand; so the carbon layer presented a typical morphology of the intumescent char layer. It is well known that the char layer can block the transfer of oxygen and heat, which decreased the combustion intensity of materials (Chen et al., 2016; Chen et al., 2017c; Li et al., 2018; Guo et al., 2019; Xu et al., 2019). From Table 4, the char yield of RPUF/MAPP-1000 

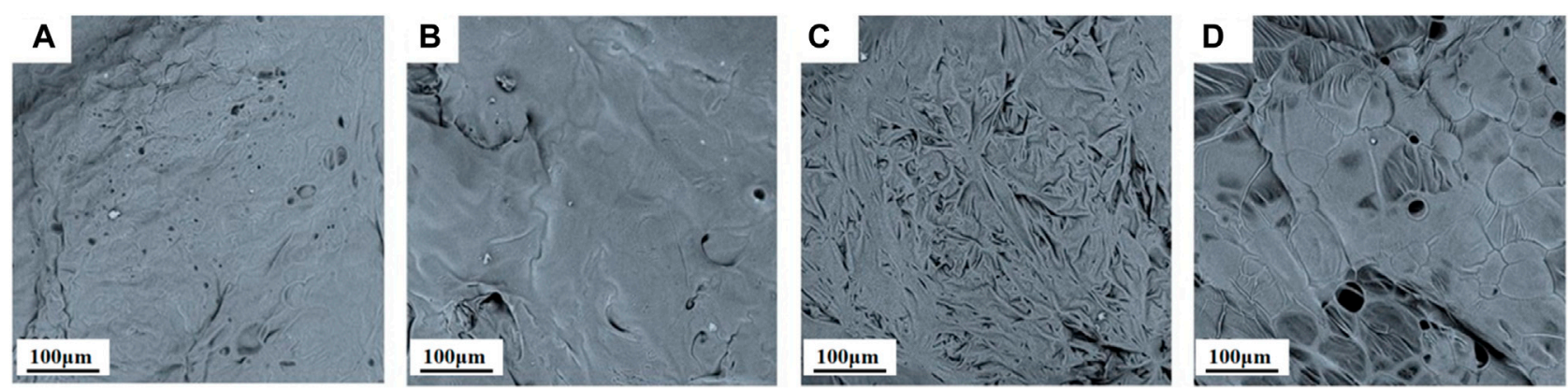

FIGURE 7 | SEM micro-morphologies of RPUF/APP-30 (A), RPUF/MAPP-30 (B), RPUF/APP-1000 (C), and RPUF/MAPP-1000 (D).

reached $36.5 \%$ at $400 \mathrm{~s}$. The results indicated that MAPP- 1000 with a high degree of polymerization was more conducive to promoting the formation of the intumescent char layer with higher thermal stability.

Figure 7 shows the SEM images of the residual char of different flame retardant RPUF composites after cone calorimetry. As shown in Figures 7A,C, the surface of RPUF/ APP carbon residue was wrinkled and uneven, and there were obvious pores and cracks on the surface. This was caused by the release of a large number of volatile gases during the combustion process of the material, which weakened the protective effect of the carbon layer on the matrix and increased the combustion intensity of RPUF/APP (Zhang et al., 2020c). As can be seen from Figures 7B,D, both RPUF/MAPP-1000 and RPUF/MAPP-30 exhibit a closed bubble structure, which is the characteristic morphology of the intumescent flame retardant systems (Zhang et al., 2021). It showed that modified APP was conducive to promoting the formation of the expanded carbon layer, thus inhibiting the exchange of heat and flammable gas, and effectively preventing further degradation of the matrix. It was found that the higher the degree of APP polymerization, the higher the viscosity at high temperature, and the gas is not easy to break through the carbon layer and overflow (Yuan et al., 2021). Therefore, the bubble structure is obvious and the expansion height of the char layer was high. On the other hand, the silicon component in PCOC could increase the strength of the char layer, which favored the formation of a complete char layer (Chen et al., 2019c). Compared with RPUF/MAP-30, the pore structure of the RPUF/MAP-1000 carbon layer was more obvious. The RPUF/ MAPP-1000 carbon layer has a honeycomb-like complete and continuous pore structure. It was possible that the carbon layer covers small molecules such as $\mathrm{NH}_{3}$ produced during the combustion of MAPP, which expanded at high temperatures to form honeycomb-like bubbles (Pan et al., 2020). This structure could provide a good insulation effect and prevent the exchange of material and heat between the flame and the foam, thus improving the flame retardant performance of the material. From the above comprehensive analysis of char residue morphologies, it can be deduced that the combination of phosphorus-containing organosilicon compounds (PCOCs) and APP can effectively improve the charring ability of the
TABLE 5 | Mechanical properties of FR-RPUFs.

\begin{tabular}{lcc} 
Samples & Density $\mathbf{( k g / \mathbf { m } ^ { \mathbf { 3 } } )}$ & Compressive strength (MPa) \\
\hline Pure RPUF & 23.9 & 0.075 \\
RPUF/APP-30 & 30.8 & 0.083 \\
RPUF/APP-1000 & 31.1 & 0.067 \\
RPUF/MAPP-30 & 32.3 & 0.068 \\
RPUF/MAPP-1000 & 28.1 & 0.066 \\
RPUF/25MAPP-1000 & 30.1 & 0.055 \\
RPUF/30MAPP-1000 & 32.2 & 0.039
\end{tabular}

RPUF, as well as help to form intumescent, continuous, and dense char layers with closed cells. The char in the form of these structures offers a good shield and protective effect during combustion.

\section{The Mechanical Properties of Pure RPUF and RPUF Composites}

The compressive strength test has been performed to study the effect of APP and MAPP on mechanical properties of RPUF. After modification, APP-30 and APP-1000 have significantly different influences on RPUF density. As can be seen from Table 5, the density of RPUF/MAPP-1000 $\left(28.1 \mathrm{~kg} / \mathrm{m}^{3}\right)$ decreased by $9.6 \%$ compared with RPUF/APP-1000 $(31.1 \mathrm{~kg} /$ $\left.\mathrm{m}^{3}\right)$, while the density of RPUF/MAPP-30 $\left(32.3 \mathrm{~kg} / \mathrm{m}^{3}\right)$ increased by $4.9 \%$ compared with RPUF/APP-30 $\left(30.8 \mathrm{~kg} / \mathrm{m}^{3}\right)$. Studies have shown that free foaming can lead to uneven foam density, and the addition of flame retardants can affect the foaming rate of matrix. Mechanical properties are important parameters in the application of materials (Xiong et al., 2019; Chen et al., 2020b; Wang et al., 2020). It could be seen that the compressive strength of the sample changed after MAPP-30 or MAPP-1000 was added to RPUF. Moreover, the compressive strength of RPUF/MAPP-30 (0.068 MPa) and RPUF/MAPP$1000(0.066 \mathrm{MPa})$ showed a slight difference. In summary, the addition of MAPP-1000 has little effect on the mechanical properties of composite materials and does not affect the application of composite materials. However, the compressive strength of RPUF/MAPP-1000 composites significantly decreased with increasing MAPP-1000 content. 


\section{CONCLUSION}

In this work, PCOC was used to modify APP with different polymerization degrees (APP-30 and APP-1000), and MAPP1000 and MAPP-30 were obtained. The influence of APP with different degrees of polymerization and modified APP on the flame retardant properties of RPUF was studied. Moreover, the effect of the content of MAPP-1000 on the flame retardant properties of the material was studied. The thermal, flameretardant properties and mechanical properties of pure RPUF and RPUF composites with APP or MAPP were investigated. TGA results showed that the residual char at $800^{\circ} \mathrm{C}$ of MAPP-30 and MAPP-1000 were $21.8 \mathrm{wt} \%$ and $22.0 \mathrm{wt} \%$, which were obviously higher than APP-30 (10.8 wt\%) and APP-1000 (10.9 wt $\%$ ), respectively. The $T_{\mathrm{d}, 5 \%}, T_{\max }$, and char yield of MAPP-1000 were higher than that of MAPP-30, indicating that MAPP-1000 had a better thermal stability. The LOI values of RPUF composites containing APP or MAPP were similar. With the increase in the addition of MAPP-1000, the LOI values of RPUF composites containing MAPP-1000 had a slight increase. The LOI values of RPUF/25MAPP-1000 and RPUF/30MAPP-1000 were both $24.9 \%$. In addition, the results of the horizontal burning test show that RPUF/APP systems and RPUF/MAPP systems all reached HF-1 rating. The cone calorimeter test results exhibited that no matter the degree of polymerization of MAPP is 30 or 1000, they both had greater charring ability and better flame-retardant properties than unmodified APP. PCOC effectively improved the charring ability of the RPUF by combining with APP. In addition, the presence of silicon in PCOC can improve the stability of the char layer at high temperatures. The char layer offers a good shield and protective effect during combustion. The residual char yield of RPUF/MAPP-30 (37.3\%) and RPUF/MAPP-1000 (36.5\%) were both significantly higher than RPUF/APP-30

\section{REFERENCES}

Bhoyate, S., Ionescu, M., Kahol, P. K., Chen, J., Mishra, S. R., and Gupta, R. K. (2018). Highly Flame-Retardant Polyurethane Foam Based on Reactive Phosphorus Polyol and Limonene-Based Polyol. J. Appl. Polym. Sci. 135 (21), 46224. doi:10.1002/app.46224

Bhoyate, S., Ionescu, M., Kahol, P. K., and Gupta, R. K. (2019). Castor-oil Derived Nonhalogenated Reactive Flame-Retardant-Based Polyurethane Foams with Significant Reduced Heat Release Rate. J. Appl. Polym. Sci. 136 (13), 47276. doi:10.1002/app.47276

Camino, G., Costa, L., and Martinasso, G. (1989). Intumescent Fire-Retardant Systems. Polym. Degrad. Stab. 23 (4), 359-376. doi:10.1016/0141-3910(89)90058-x

Chen, X., Li, J., and Gao, M. (2019). Thermal Degradation and Flame Retardant Mechanism of the Rigid Polyurethane Foam Including Functionalized Graphene Oxide. Polymers 11 (1), 78. doi:10.3390/polym11010078

Chen, Y. J., Wang, W., Liu, Z. Q., Yao, Y. Y., and Qian, L. J. (2017). Synthesis of a Novel Flame Retardant Containing Phosphazene and Triazine Groups and its Enhanced Charring Effect in Poly(lactic Acid) Resin[J]. J. Appl. Polym. Sci. 134 (13), 44660. doi:10.1002/app.44660

Chen, Y. J., Wu, X. D., and Qian, L. J. (2020). Flame-retardant Behavior and Protective Layer Effect of Phosphazene-Triazine Bi-group Flame Retardant on Polycarbonate[J]. J. Appl. Polym. Sci. 137 (46), e49523. doi:10.1002/app.49523

Chen, Y., Li, L., Qi, X., and Qian, L. (2019). The Pyrolysis Behaviors of PhosphorusContaining Organosilicon Compound Modified APP with Different Polyether
(22.8\%) and RPUF/APP-1000 (24.9\%). The peak heat release rate value of RPUF/MAPP-30 was $29.9 \%$ lower than that of RPUF/APP-30, and the drop of RPUF/MAPP-1000 was $50.9 \%$ compared to RPUF/APP-1000. Moreover, the total heat release of RPUF/MAPP-1000 $\left(9.7 \mathrm{MJ} / \mathrm{m}^{2}\right)$ was much lower than that of RPUF/MAPP-30 $\left(11.3 \mathrm{MJ} / \mathrm{m}^{2}\right)$. It was worth noting that MAPP1000 has the best flame-retardant properties among all RPUF composites, and the amount of MAPP was only $20 \%$. The compressive strength test results showed that the addition of MAPP-1000 has little effect on the mechanical properties of composite materials and does not affect the application of composite materials. However, the compressive strength of RPUF/MAPP-1000 composites significantly decreased with increasing MAPP-1000 content. In summary, MAPP-1000 was a high-performance flame-retardant system with outstanding charring ability, and the RPUF/MAPP-1000 composite had the best comprehensive performance when the MAP-1000 content was $20 \%$.

\section{DATA AVAILABILITY STATEMENT}

The original contributions presented in the study are included in the article/supplementary material; further inquiries can be directed to the corresponding author.

\section{AUTHOR CONTRIBUTIONS}

CY was involved in conceptualization, writing-original draft preparation, and writing-review and editing; CY and SS were involved in the methodology, software, and supervision. Both authors have read and agreed to the published version of the manuscript.

Segments and Their Flame Retardant Mechanism in Polyurethane Foam. Composites B: Eng. 173, 106784. doi:10.1016/j.compositesb.2019.04.045

Chen, Y., Li, L., Wang, W., and Qian, L. (2017). Preparation and Characterization of Surface-Modified Ammonium Polyphosphate and its Effect on the Flame Retardancy of Rigid Polyurethane Foam. J. Appl. Polym. Sci. 134 (40), 45369. doi:10.1002/app.45369

Chen, Y., Li, L., and Wu, X. (2020). Construction of an Efficient Ternary Flame Retardant System for Rigid Polyurethane Foam Based on Bi-phase Flame Retardant Effect. Polym. Adv. Technol. 31 (12), 3202-3210. doi:10.1002/pat.5045

Chen, Y., Li, L., Xu, L., and Qian, L. (2018). Phosphorus-containing Silica GelCoated Ammonium Polyphosphate: Preparation, Characterization, and its Effect on the Flame Retardancy of Rigid Polyurethane Foam. J. Appl. Polym. Sci. 135, 46334. doi:10.1002/app.46334

Chen, Y., Mao, X., Qian, L., and Yang, C. (2016). Flammability and Anti-dripping Behaviors of Polylactide Composite Containing Hyperbranched Triazine Compound. Integrated Ferroelectrics 172 (1), 10-24. doi:10.1080/10584587.2016.1175257

Chen, Y., Wang, W., Qiu, Y., Li, L., Qian, L., and Xin, F. (2017). Terminal Group Effects of Phosphazene-Triazine Bi-group Flame Retardant Additives in Flame Retardant Polylactic Acid Composites. Polym. Degrad. Stab. 140, 166-175. doi:10.1016/j.polymdegradstab.2017.04.024

Chen, Y., Xu, L., Wu, X., and Xu, B. (2019). The Influence of Nano ZnO Coated by Phosphazene/triazine Bi-group Molecular on the Flame Retardant Property and Mechanical Property of Intumescent Flame Retardant Poly (Lactic Acid) Composites. Thermochim. Acta 679, 178336. doi:10.1016/ j.tca.2019.178336 
Deodhar, S., Shanmuganathan, K., Fan, Q., Wilkie, C. A., Costache, M. C., Dembsey, N. A., et al. (2011). Calcium Carbonate and Ammonium Polyphosphate-Based Flame Retardant Composition for Polypropylene. J. Appl. Polym. Sci. 120 (3), 1866-1873. doi:10.1002/app.32510

Guo, C., Xin, F., Zhai, C., and Chen, Y. (2019). Flammability and thermal Properties of Modified Carbon Nanotubes in Poly(lactic Acid). J. Thermoplastic Compos. Mater. 32 (8), 1107-1122. doi:10.1177/0892705718785678

Hazer, S., Coban, M., and Aytac, A. (2019). Effects of Ammonium Polyphosphate and Triphenyl Phosphate on the Flame Retardancy, thermal, and Mechanical Properties of Glass Fiber-Reinforced PLA/PC Composites. Fire Mater. 43 (3), 277-282. doi:10.1002/fam.2696

Jing, J., Zhang, Y., Fang, Z.-P., and Wang, D.-Y. (2018). Core-shell Flame Retardant/graphene Oxide Hybrid: a Self-Assembly Strategy towards Reducing Fire hazard and Improving Toughness of Polylactic Acid. Composites Sci. Tech. 165, 161-167. doi:10.1016/j.compscitech.2018.06.024

Levchik, S. V., and Weil, E. D. (2004). Thermal Decomposition, Combustion and Fire-Retardancy of Polyurethanes-A Review of the Recent Literature. Polym. Int. 53, 1585-1610. doi:10.1002/pi.1314

Li, L., Chen, Y., Wu, X., Xu, B., and Qian, L. (2019). Bi-phase Flame-retardant Effect of Dimethyl Methylphosphonate and Modified Ammonium Polyphosphate on Rigid Polyurethane Foam. Polym. Adv. Technol. 30, 2721-2728. doi:10.1002/pat.4702

Li, L., Mao, X., Ju, R., Chen, Y., and Qian, L. (2018). Synergistic Effect of OrganoMontmorillonite on Intumescent Flame-Retardant PLA. Ferroelectrics 527, 25-36. doi:10.1080/00150193.2018.1450045

Li, Q., Wang, J., Chen, L., Shi, H., and Hao, J. (2019). Ammonium Polyphosphate Modified with $\beta$-cyclodextrin Crosslinking Rigid Polyurethane Foam: Enhancing thermal Stability and Suppressing Flame Spread. Polym. Degrad. Stab. 161, 166-174. doi:10.1016/j.polymdegradstab.2019.01.024

Liu, Y., He, J., and Yang, R. (2016). The Preparation and Properties of FlameRetardant Polyisocyanurate-Polyurethane Foams Based on Two DOPO Derivatives. J. Fire Sci. 34 (5), 431-444. doi:10.1177/0734904116662667

Pan, Y.-T., Zhang, Z., and Yang, R. (2020). The Rise of MOFs and Their Derivatives for Flame Retardant Polymeric Materials: A Critical Review. Composites Part B: Eng. 199, 108265. doi:10.1016/j.compositesb.2020.108265

Qin, Z., Li, D., Zhang, W., and Yang, R. (2015). Surface Modification of Ammonium Polyphosphate with Vinyltrimethoxysilane: Preparation, Characterization, and its Flame Retardancy in Polypropylene. Polym. Degrad. Stab. 119, 139-150. doi:10.1016/j.polymdegradstab.2015.05.012

Sai, T., Ran, S., Guo, Z., Yan, H., Zhang, Y., Wang, H., et al. (2021). Transparent, Highly Thermostable and Flame Retardant Polycarbonate Enabled by Rod-like Phosphorous-Containing Metal Complex Aggregates. Chem. Eng. J. 409, 128223. doi:10.1016/j.cej.2020.128223

Shaw, S., Blum, A., Weber, R., Kannan, K., and Birnbaum, L. S. (2010). Halogenated Flame Retardants: Do the Fire Safety Benefits Justify the Risks? [J]. Rev. Environ. Health 25 (4), 261-306. doi:10.1515/reveh.2010.25.4.261

Sypaseuth, F. D., Gallo, E., Çiftci, S., and Schartel, B. (2017). Polylactic Acid Biocomposites: Approaches to a Completely green Flame Retarded Polymer. E-Polymers 17 (6), 449-462. doi:10.1515/epoly-2017-0024

Wang, S., Wang, X., Wang, X., Li, H., Sun, J., Sun, W., et al. (2020). Surface Coated Rigid Polyurethane Foam with Durable Flame Retardancy and Improved Mechanical Property. Chem. Eng. J. 385, 123755. doi:10.1016/j.cej.2019.123755

Watanabe, M., Watanabe, M., Narita, N., Sakurai, M., and Suzuki, H. (2000). Preparation of Ammonium Polyphosphate Form II from the System of Ammonium Orthophosphate-Urea. Bcsj 73 (1), 115-119. doi:10.1246/bcsj.73.115

Wu, C., Wang, X., Zhang, J., Cheng, J., and Shi, L. (2019). Microencapsulation and Surface Functionalization of Ammonium Polyphosphate via In-Situ Polymerization and Thiol-Ene Photograted Reaction for Application in Flame-Retardant Natural Rubber. Ind. Eng. Chem. Res. 58 (37), 17346-17358. doi:10.1021/acs.iecr.9b02464

Xi, W., Qian, L., Huang, Z., Cao, Y., and Li, L. (2016). Continuous Flame-Retardant Actions of Two Phosphate Esters with Expandable Graphite in Rigid Polyurethane Foams. Polym. Degrad. Stab. 130, 97-102. doi:10.1016/j.polymdegradstab.2016.06.003

Xiong, Z., Zhang, Y., Du, X., Song, P., and Fang, Z. (2019). Green and Scalable Fabrication of Core-Shell Biobased Flame Retardants for Reducing Flammability of Polylactic Acid. ACS Sust. Chem. Eng. 7 (9), 8954-8963. doi:10.1021/acssuschemeng.9b01016

Xu, L., Wu, X., Li, L., and Chen, Y. (2019). Synthesis of a Novel Polyphosphazene/ triazine Bi-group Flame Retardant In Situ Doping Nano Zinc Oxide and its
Application in Poly (Lactic Acid) Resin. Polym. Adv. Technol. 30 (6), 1375-1385. doi:10.1002/pat.4570

Xu, W., Wang, G., and Zheng, X. (2015). Research on Highly Flame-Retardant Rigid PU Foams by Combination of Nanostructured Additives and Phosphorus Flame Retardants. Polym. Degrad. Stab. 111, 142-150. doi:10.1016/ j.polymdegradstab.2014.11.008

Yang, H., Yu, B., Song, P., Maluk, C., and Wang, H. (2019). Surface-coating Engineering for Flame Retardant Flexible Polyurethane Foams: A Critical Review. Composites Part B: Eng. 176, 107185. doi:10.1016/j.compositesb.2019.107185

Yang, M. S., Liu, Y., Lin-Kai, L. I., and Ding, J. (2009). Synthesis of Hexaphenylamine Cyclotriphosphazene Flame Retardant and its Application in Epoxy Molding Compound for Large-Scale Integrated Circuit Packaging[J]. China Plastics Industry 37 (8), 61-65.

Yang, R., Hu, W., Xu, L., Song, Y., and Li, J. (2015). Synthesis, Mechanical Properties and Fire Behaviors of Rigid Polyurethane Foam with a Reactive Flame Retardant Containing Phosphazene and Phosphate. Polym. Degrad. Stab. 122, 102-109. doi:10.1016/j.polymdegradstab.2015.10.007

Yang, R., Wang, B., Han, X., Ma, B., and Li, J. (2017). Synthesis and Characterization of Flame Retardant Rigid Polyurethane Foam Based on a Reactive Flame Retardant Containing Phosphazene and Cyclophosphonate. Polym. Degrad. Stab. 144, 62-69. doi:10.1016/j.polymdegradstab.2017.08.008

Yuan, Y., Pan, Y.-T., Zhang, Z., Zhang, W., Li, X., and Yang, R. (2021). Nickle Nanocrystals Decorated on Graphitic Nanotubes with Broad Channels for Fire hazard Reduction of Epoxy Resin. J. Hazard. Mater. 402, 123880. doi:10.1016/ j.jhazmat.2020.123880

Yuan, Y., Yang, H., Yu, B., Shi, Y., Wang, W., Song, L., et al. (2016). Phosphorus and Nitrogen-Containing Polyols: Synergistic Effect on the Thermal Property and Flame Retardancy of Rigid Polyurethane Foam Composites. Ind. Eng. Chem. Res.eEngineering Chem. Res. 55 (41), 10813-10822. doi:10.1021/ acs.iecr.6b02942

Yuan, Y., Yang, H., Yu, B., Shi, Y., Wang, W., Song, L., et al. (2016). Phosphorus and Nitrogen-Containing Polyols: Synergistic Effect on the Thermal Property and Flame Retardancy of Rigid Polyurethane Foam Composites. Ind. Eng. Chem. Res. 55 (41), 10813-10822. doi:10.1021/acs.iecr.6b02942

Zhang, K., Hong, Y., Wang, N., and Wang, Y. (2018). Flame Retardant Polyurethane Foam Prepared from Compatible Blends of Soybean Oil-Based Polyol and Phosphorus Containing Polyol. J. Appl. Polym. Sci. 135 (5), 45779. doi:10.1002/app.45779

Zhang, Y., Jing, J., Liu, T., Xi, L., Sai, T., Ran, S., et al. (2021). A Molecularly Engineered Bioderived Polyphosphate for Enhanced Flame Retardant, UVBlocking and Mechanical Properties of Poly(lactic Acid). Chem. Eng. J. 411, 128493. doi:10.1016/j.cej.2021.128493

Zhang, Y., Xiong, Z., Ge, H., Ni, L., Zhang, T., Huo, S., et al. (2020). CoreShell Bioderived Flame Retardants Based on Chitosan/Alginate Coated Ammonia Polyphosphate for Enhancing Flame Retardancy of Polylactic Acid. ACS Sust. Chem. Eng. 8 (16), 6402-6412. doi:10.1021/acssuschemeng.0c00634

Zhang, Z., Han, Z., Pan, Y.-T., Li, D., Wang, D.-Y., and Yang, R. (2020). Dry Synthesis of Mesoporous Nanosheet Assembly Constructed by Cyclomatrix Polyphosphazene Frameworks and its Application in Flame Retardant Polypropylene. Chem. Eng. J. 395, 125076. doi:10.1016/j.cej.2020.125076

Zhang, Z., Li, D., Xu, M., and Li, B. (2020). Synthesis of a Novel Phosphorus and Nitrogen-Containing Flame Retardant and its Application in Rigid Polyurethane Foam with Expandable Graphite. Polym. Degrad. Stab. 173, 109077. doi:10.1016/j.polymdegradstab.2020.109077

Zheng, Z., Liu, Y., Zhang, L., Dai, B., Yang, X., and Wang, H. (2017). Fabrication of Halogen-free Ammonium Phosphate with Two Components via a Simple Method and its Flame Retardancy in Polypropylene Composites. J. Therm. Anal. Calorim. 127 (3), 2013-2023. doi:10.1007/s10973-016-5779-x

Conflict of Interest: CY and SS were employed by Hangzhou Zhihe New Material Co., Ltd.

Copyright (๑) 2021 Yang and Shao. This is an open-access article distributed under the terms of the Creative Commons Attribution License (CC BY). The use, distribution or reproduction in other forums is permitted, provided the original author(s) and the copyright owner(s) are credited and that the original publication in this journal is cited, in accordance with accepted academic practice. No use, distribution or reproduction is permitted which does not comply with these terms. 